\title{
Domain Evolution and Polarization of Continuously Graded Ferroelectric Films
}

\author{
(Dedicated to Prof A. Khachaturian on his 75th Birthday)
}

\author{
Alexander Roytburd ${ }^{\mathrm{a}}$ and Victor Roytburd ${ }^{\mathrm{b} *}$ \\ ${ }^{a}$ Department of Materials Science and Engineering University of Maryland, \\ College Park, MD 20\%42; ${ }^{\mathrm{b}}$ Department of Mathematical Sciences, \\ Rensselaer Polytechnic Institute, Troy, NY 12180
}

\begin{abstract}
A thermodynamic analysis of graded ferroelectric films demonstrates that in the equilibrium state the films are subdivided into a single-domain band and a polydomain band which consists of wedge-shape domains. Polarization under an external electrostatic field proceeds through an inter-band boundary movement due to growth or shrinkage of the wedge domains.

It is shown how the domain structure and evolution are determined by the principal characteristics of the film: the distribution of the spontaneous polarization and dielectric constant. Graded films exhibit a sharp increase of polarization with the field for weak fields, with a drop of the dielectric constant when the field is increasing.

A general approach to finding the dependence of the displacement and the wedgedomain shape on the field as well as analytical solutions for the $p^{4}$ Landau-Devonshire and parabolic potentials are presented.
\end{abstract}

Keywords: graded ferroelectrics, ferroelectric films, ferroelectric domains

${ }^{*}$ Corresponding author. Email: roytbv@rpi.edu; Phone: 703-292-2189; Fax: 703-292-9032 


\section{Introduction}

Graded ferroelectric films and multilayers are highly sensitive to the electric field and temperature changes; this gives rise to their use for microelectronic applications [1]. However, after many years of theoretical and experimental studies there is no definitive understanding of mechanisms that determine special functional properties of graded ferroelectrics. Most of the theoretical work in this area relates fundamental properties of graded ferroelectrics to the short-range polarization self-interactions, which are represented by the gradient energy term in the Landau-Devonshire potential. These interactions are essential if the gradients of polarization are comparable to those in the domain walls. The effects of smaller gradients may be important in the absence of strong long-range electrostatic interactions, for example, in ferroelectrics with high concentration of free charges [2].

A different approach was presented in [3] where the dominant role of the long-range interactions was suggested. It has been shown that minimization of electrostatic energy of a graded multilayer results in the formation of a special wedge-domain structure. Polarization of multilayers proceeds through movement of domain walls with progressive transformation of polydomain layers into the single-domain ones. The theory of [3] provides the principal explanation of dielectric properties of graded multilayers, in particular it explains a dramatic increase of the dielectric constant with the field decrease.

This thermodynamic theory does not take into account short-range interactions, thus neglecting the domain walls energy. However the comparison of the theoretical results with phase-field modeling that does include all interactions shows a good qualitative agreement [4]. It confirms that electrostatic interactions do dominate formation and evolution of the domain structure in graded ferroelectrics.

In this paper we extend the concept of wedge domains to continuously graded ferroelectric films. We will consider the equilibrium state of a ferroelectric film, with its parameters (spontaneous polarization, electric susceptibility, etc.) changing continuously throughout the film thickness. For simplicity we treat the case of a uniaxial ferroelectric.

\section{Theory}

The equilibrium state of a single-domain film is determined by the minimum of the Gibbs free energy

$$
F(P(X))=\int_{0}^{L}\left[f(P(X), X)+\left(1 / 2 \varepsilon_{0}\right)(P(X)-\bar{P})^{2}-E P(X)\right] d X,
$$

where $L$ is the film thickness; $P(X)$ is the polarization in the layer $X ; \bar{P}$ is the average polarization, $\bar{P}=L^{-1} \int_{0}^{L} P(X) d X ; E$ is the external electrostatic field generated by the electrodes under the potential $U=E L$ (the short-circuit condition is being considered). The free energy density $f(P)$ is given by the Landau-Devonshire potential; it describes the microscopic energy of the ferroelectric for the given value of the order parameter $P$. This potential determines spontaneous polarization in each layer $P_{0}(X)$. To avoid complications due to the elastic interaction between the layers we consider a film clamped by substrate; thus we use the Landau-Devonshire potential that is normalized to include the elastic effect 
of the substrate. The other two terms in (1) represent the macroscopic contribution of the electrostatic field to the Gibbs free energy: the electrostatic energy of the interaction between the layers, and the work of the electric field.

For the polydomain film, which is an alternation of two domains with polarizations of opposite directions, the macroscopic terms preserve their form while the microscopic component becomes a weighted average of the single-domain energies:

$$
F(P)=\int_{0}^{L}\left[(1-\beta) f\left(P_{1}(X), X\right)+\beta f\left(P_{2}(X), X\right)+\left(1 / 2 \varepsilon_{0}\right)(\tilde{P}-\bar{P})^{2}-E \tilde{P}\right] d X
$$

where $\beta(X)$ and $1-\beta(X)$ are the fractions of the domains with the opposite direction polarizations $P_{2}$ and $P_{1}$. The macroscopic polarization in the layer $X$ is given by $\tilde{P}=$ $(1-\beta) P_{1}+\beta P_{2}$ while $\bar{P}$ here is the average of $\tilde{P}$.

We will show that for the ferroelectric film the equilibrium state has a band structure, with alternating bands of single-domain layers and of polydomain layers. This band structure is completely determined by the microscopic potential $f$ and by the applied field $E$. Under variation of the external field the film polarization changes due to the motion of the boundary between the bands, which in its turn is caused by the single domain $\rightleftarrows$ polydomain transformations of the layers.

To analyze the simplest band architecture we consider the spontaneous polarization profile $P_{0}(X)$ which increases monotonically from $P_{0}(0)=\mathcal{P}_{0}$ on one surface of the film to $P_{0}(L)$ on the other. We will prove that in this case the film will subdivide into two bands: a single-domain band at $0<X<\mathcal{L}$, and a polydomain one at $\mathcal{L}<X<L$, Fig. 1 . In the dimensionless form the Gibbs free energy for the two-band film is as follows:

$$
\begin{aligned}
\mathcal{F}(p, \mathcal{E}) & =F /\left(L \mathcal{P}_{0}^{2} / \varepsilon_{0}\right)=\mathcal{F}_{1}+\mathcal{F}_{2}=\int_{0}^{\xi}\left[\phi(p, x)-\mathcal{E} p(x)+(p(x)-\bar{p})^{2} / 2\right] d x \\
& +\int_{\xi}^{1}\left[(1-\beta(x)) \phi\left(p_{1}(x)\right)+\beta(x) \phi\left(p_{2}(x)\right)-\mathcal{E} \tilde{p}(x)+(\tilde{p}(x)-\bar{p})^{2} / 2\right] d x
\end{aligned}
$$

where $x=X / L, \xi=\mathcal{L} / L, \phi=f /\left(\mathcal{P}_{0}^{2} / \varepsilon_{0}\right), \mathcal{E}=E /\left(\mathcal{P}_{0} / \varepsilon_{0}\right), p(x)=P(x L) / \mathcal{P}_{0}, p_{1}(x)=$ $P_{1}(x L) / \mathcal{P}_{0}, p_{2}(x)=P_{2}(x L) / \mathcal{P}_{0}, \tilde{p}(x)=(1-\beta(x)) p_{1}(x)+\beta(x) p_{2}(x), \bar{p}=\int_{0}^{\xi} p(x) d x+$ $\int_{\xi}^{1} \tilde{p}(x) d x$. Thus the Gibbs free energy depends on $\xi$ and on the following functional parameters: $p(x), p_{1}(x), p_{2}(x)$, and $\beta(x)$.

For the polarization fields $p(x), p_{1}(x)$, and $p_{2}(x)$ to realize the minimum of the Gibbs free energy they have to satisfy the following variational equations. First of all, in the single-domain band $(x<\xi)$,

$$
\delta \mathcal{F}_{1} / \delta p=\partial \phi / \partial p-\mathcal{E}+p(x)-\bar{p}=0 .
$$

In the polydomain band $(\xi<x<1)$

$$
\delta \mathcal{F}_{2} / \delta \beta=-\phi\left(p_{1}, x\right)+\phi\left(p_{2}, x\right)+E\left(p_{1}-p_{2}\right)-(\tilde{p}(x)-\bar{p})\left(p_{1}-p_{2}\right)=0 .
$$

Variations with respect to $p_{1}$ and $p_{2}$ yield

$$
\begin{aligned}
& \delta \mathcal{F}_{2} / \delta p_{1}=(1-\beta)[-E+\tilde{p}(x)-\bar{p}]=0 \\
& \delta \mathcal{F}_{2} / \delta p_{2}=\beta\left[\partial \phi / \partial p_{2}-E+\tilde{p}(x)-\bar{p}\right]=0
\end{aligned}
$$


In view of (5)-(7),

$$
\partial \phi / \partial p_{1}=\partial \phi / \partial p_{2}=\left(\phi\left(p_{2}, x\right)-\phi\left(p_{1}, x\right)\right) /\left(p_{2}-p_{1}\right)=\mathcal{E}-\tilde{p}(x)+\bar{p}
$$

Geometrically these equations mean that the tangents to $\phi$ at $p_{1}$ and $p_{2}$ coincide. Since $\phi$ is the symmetric two-well potential with equal minima at $p(x)= \pm p_{0}(x)$, the only values $p_{1}$ and $p_{2}$ for which equations in (8) hold are

$$
p_{1}=-p_{2}=p_{0} .
$$

From the condition that the variational derivative of the Gibbs free energy with respect to $\xi$ is zero, it is easy to show that the polarization should be continuous through the interface $x=\xi$ and therefore $\beta(\xi)=0$. It immediately yields that for $\xi<x<1$,

$$
\tilde{p}(x)=(1-2 \beta(x)) p_{0}(x)=(1-2 \beta(\xi)) p_{0}(\xi)=p_{0}(\xi) .
$$

Moreover, the common value of all the equations in (8) is 0 ; in particular, $\mathcal{E}-\tilde{p}(x)+\bar{p}=0$, i.e., for $\xi<x<1$,

$$
\mathcal{D}(\mathcal{E})=\mathcal{E}+\bar{p}=\mathcal{E}+\int_{0}^{\xi} p(x) d x+(1-\xi) p_{0}(\xi)=p_{0}(\xi) .
$$

Given $\mathcal{D}=p_{0}(\xi)$, the fraction $\beta$ is found explicitly from $(10)$ :

$$
\beta=1 / 2-\mathcal{D} / 2 p_{0}(x)
$$

The expression (11) for $\mathcal{D}$ is deceivingly simple. What is hidden in this expression is that the inter-band boundary position $\xi$ is related to $\mathcal{E}$ and that $p$ actually depends on $\mathcal{E}$, $p=p(x ; \xi(\mathcal{E}))$ being a solution of Eq. (4), which in view of (11) takes the form

$$
\partial \phi / \partial p+p(x)-p_{0}(\xi)=0 .
$$

However, if $\xi$ is taken as a departure point instead of $\mathcal{E}$ then $\mathcal{D}=\mathcal{E}+\bar{p}(\mathcal{E})$ versus $\mathcal{E}$ can be found quite easily as a parametric curve. Suppose $\xi$ is the optimal position of the band interface for a given $\mathcal{E}$. Then

$$
p(x)=\left\{\begin{array}{c}
p(x ; \xi), \quad x<\xi \\
p_{0}(\xi), \quad 1>x>\xi
\end{array}\right.
$$

where $p(x ; \xi)$ is a solution of Eq. (13), which for an arbitrary $\phi(p(x), x)$ can be easily solved numerically, say, by a Newton's method. Then the field is determined from

$$
\mathcal{E}(\xi)=\mathcal{D}-\bar{p}=p_{0}(\xi)-\int_{0}^{\xi} p(x ; \xi) d x-(1-\xi) p_{0}(\xi)=\xi p_{0}(\xi)-\int_{0}^{\xi} p(x ; \xi) d x
$$

Eqs. (11)-(15) define $\mathcal{D}$ versus $\mathcal{E}$ as a parametric curve.

So far we considered the case of two bands, i.e., when the band interface $\xi<1$. If $\xi=1$, then the free energy is given by (1) and in the dimensionless form the variational problems becomes as follows

$$
\mathcal{F}_{1}(p, \mathcal{E})=\int_{0}^{1}\left[\phi(p, x)-\mathcal{E} p(x)+(p(x)-\bar{p})^{2} / 2\right] d x \rightarrow \min
$$


Then $p(x)$ is found from Eq. (4), which in this case yields

$$
\partial \phi / \partial p-\mathcal{D}+p(x)=0
$$

The corresponding electrical field is then equal to

$$
\mathcal{E}=\mathcal{D}-\int_{0}^{1} p(x ; \mathcal{D}) d x
$$

This latter formula gives the relation between $\mathcal{E}$ and $\mathcal{D}$ for the one-band case.

In the next sections we present analytical solutions for two important cases, a simple form of the Landau-Devonshire potential, and its parabolic approximation.

\section{Landau-Devonshire potential}

If $\phi$ is a $p^{4}$ Landau-Devonshire potential

$$
\phi(p, x)=A(x)\left(-\frac{p^{2}}{2}+\frac{p^{4}}{4 p_{0}^{2}(x)}\right), \quad A(x)=\frac{1}{2(\varepsilon(x)-1)}
$$

then Eq. (13) is cubic and can be solved explicitly via Cardano's formulae. Fig. 2 illustrates the dependence of the displacement on the field for three spontaneous polarization profiles. The curves for the single-domain films are shown for comparison. It is clear that the polydomain band is responsible for the sharp increase of the dielectric constant at low fields. This results from the absence of the field in the polydomain band and its concentration in the single-domain one. This effect can be interpreted as the decrease of the effective film thickness with the field. The apparent singularity at $\mathcal{E}=l$ is the consequence of the singledomain band thickness tending to zero. In actual graded films the layers with minimum spontaneous polarization which remains in the single-domain state have finite thickness and the dielectric has a large however finite magnitude.

In Fig. 3 we present the wedge-domain shape evolutions that correspond to the polarization of graded film from Fig. 2. This domain shapes are calculated according to Eq. (12) that can be restated as follows

$$
\beta=1 / 2-p_{0}(\xi) / 2 p_{0}(x)
$$

The dependence of $\beta$ on the coordinate $x$ describes how the thickness of the wedge domain increases with the distance from the band interface; at the interface, $x=\xi$, the thickness goes to zero. The shape of the domain tip depends on the characteristics of the graded film, in particular on the polarization profile $p_{0}(x)$. Under the weak field, the domain tip has a rounded shape for $p_{0}(x) \sim x^{1 / 2}$, a cusp for $p_{0}(x) \sim x^{2}$, and makes an acute angle for $p_{0}(x) \sim x$. As the filed increases the tip shape differences become less pronounced. Similarly to the crack propagation the domain tip shape is important for the kinetics of domain evolution. However, this issue is beyond the scope of the thermodynamic study of the present paper; it will be discussed elsewhere. 


\section{Parabolic potential}

In practically important cases of moderate gradients of spontaneous polarization, the potential is well-approximated by a quadratic function

$$
\phi=\left(p(x)-p_{0}(x)\right)^{2} / 2(\varepsilon(x)-1) .
$$

Then the solution is particularly simple:

$$
p(x)=\left\{\begin{array}{c}
(1 / \varepsilon(x)) p_{0}(x)+(1-(1 / \varepsilon(x))) p_{0}(\xi), \quad x<\xi_{0} \\
p_{0}(\xi), \quad 1>x>\xi
\end{array}\right.
$$

For $\mathcal{D}$ we get the

$$
\mathcal{D}=p_{0}(\xi)=\mathcal{E} / \int_{0}^{\xi} \frac{d x}{\varepsilon(x)}+\int_{0}^{\xi} \frac{p_{0}(x)}{\varepsilon(x)} d x / \int_{0}^{\xi} \frac{d x}{\varepsilon(x)}
$$

(a version of this formula for graded multilayers was introduced in [3]). Eq. (23) can also be restated as follows:

$$
\mathcal{E}=p_{0}(\xi) \int_{0}^{\xi} \frac{d x}{\varepsilon(x)}-\int_{0}^{\xi} \frac{p_{0}(x)}{\varepsilon(x)} d x=\int_{0}^{\xi}\left(\frac{d p_{0}(x)}{d x} \int_{0}^{x} \frac{1}{\varepsilon(s)} d s\right) d x
$$

It relates $\xi$ and $\mathcal{E}$ through the basic characteristics of the graded film, its gradient of spontaneous polarization and dielectric constant distribution.

For example, if the polarization and susceptibility are given by $p_{0}(x)=1+k x^{n}, n>0$, and $1 / \varepsilon(x)=1 / \varepsilon_{\max }\left(1+\chi x^{m}\right)$ then $\xi(\mathcal{E})$ is determined from the following equation

$$
\xi^{n+1}+\chi \xi^{n+m+1}=\left(\varepsilon_{\max } / k\right) \mathcal{E}
$$

It clearly shows that for relatively weak fields where $\xi \ll 1$ the first term in (25) dominates. Then $\xi$ as well as $\mathcal{D}$ are determined by the polarization gradient $d p_{0} / d x$, and in this approximation

$$
\xi=\left(\varepsilon_{\max } \mathcal{E}(n+1) / n k\right)^{1 /(n+1)}, \quad \mathcal{D}=1+k^{1 /(n+1)}\left[\varepsilon_{\max }(n+1) \mathcal{E} / n\right]^{n /(n+1)}
$$

In particular, if $n=1$, then $\xi=\sqrt{2 \varepsilon_{\max } \mathcal{E} / k}$ and $\mathcal{D}=1+\sqrt{2 \varepsilon_{\max } \mathcal{E} k}$.

On the other hand, the field $\mathcal{E}_{c}$ corresponding to the completely transformed state (at $\xi=1$ ) depends also on the distribution of the dielectric constant

$$
\mathcal{E}_{c}=\left(k / \varepsilon_{\max }\right)\{[n /(n+1)]+\chi[n /(m+1)(m+n+1)]\}
$$

A similar dependence of the dielectric response of the graded film on its characteristics exists for the $p^{4}$ potential and is expected for a more general form of Landau-Devonshire energy. In Fig. 4 we compare the $\mathcal{D}(\mathcal{E})$ curves for the $p^{4}$ potential with $p_{0}(x)=1+0.5 x$ and two different dielectric constant profiles (the constant is select as the average of the variable profile). It confirms the above mentioned effects of the polarization and susceptibility on the polarization curve. 


\section{Concluding remarks}

The analysis above has been focused on the two-band structure; however, it can be easily generalized to the multi-band structure. By using a non-monotonic spontaneous polarization profile it is possible to obtain multi-band wedge domain structures, with nonsmooth $\mathcal{D}(\mathcal{E})$ dependences. Our thermodynamic approach used the homogenization approximation, which is valid if the domain structure period is much smaller than the domain thickness. This corresponds to a relatively small contribution of the domain walls into the graded film thermodynamics. Since the ratio of the domain wall energy to the characteristic electrostatic energy $\left(\mathcal{P}_{0}^{2} / \varepsilon_{0}\right)$ is on the order of $\sqrt{l / L}$, where $l$ is the domain wall thickness, the homogenization is an adequate approximation for thermodynamics of the films no thinner than tens of $\mathrm{nm}$. This approximation does not take into account hysteresis effects due to the generation [5] and annihilation of domain walls during the wedge domain evolution. However the wedge domain walls are expected to be highly mobile because they deviate from the close-packed plane orientation and do not need to overcome energy barriers between discrete layers, in contrast with the graded multilayers [3]. If the complete transformation of the film to the single-domain state is not attained (in particular, for films with strong polarization gradients) then the domain evolution can be highly reversible. Thus, the thermodynamic approach of this paper provides useful insights into understanding the behavior of graded ferroelectrics. Results of this paper open possibilities of designing graded films with desirable dielectric properties.

Acknowledgments. A.R. gratefully acknowledges the support of the Israel-USA Binational Science Foundation. V.R.'s work was partially supported by the National Science Foundation, while working at the Foundation. Part of his work was done while on a sabbatical leave at the Lawrence Berkeley National Laboratory.

\section{References}

[1] J. V. Mantese and S. P. Alpay, Graded Ferroelectrics, Transpacitors and Transponents (Springer, New York, 2005)

[2] Z.-G. Ban, S. P Alpay, and J. V. Mantese, Phys. Rev. B 67, 184104 (2003)

[3] A. L. Roytburd and J. Slutsker, Appl. Phys Lett. 89, 042907 (2006).

[4] A. Artemov et al, J. Appl. Phys. 103, 074104 (2008).

[5] A. M. Bratkovsky and A. P. Levanyuk, Phys. Rev. B 66, 184109 (2002). 


\section{Figure Captions}

Figure 1: Two-band film structure.

Figure 2: The polarization curves $\mathcal{D}(\mathcal{E})$ for $A(x)=0.005$ and for three model polarization profiles: $p_{0}(x)=1+0.5 x$ (thin line); $p_{0}(x)=1+0.5 x^{2}$ (dot-dashed line); $p_{0}(x)=1+0.5 \sqrt{x}$ (dashed line). The level $\mathcal{D} \geq 1.5$ and the return (higher) curves correspond to the singledomain state.

Figure 3: Wedge shapes for the three positions of the band interface $\xi=0,1 / 3,2 / 3$, and for the three model polarization profiles: (a) $p_{0}(x)=1+0.5 x$; (b) $p_{0}(x)=1+0.5 x^{2}$; (c) $p_{0}(x)=1+0.5 \sqrt{x}$.

Figure 4: Comparison of $\mathcal{D}(\mathcal{E})$ curves for the $p^{4}$ potential with $p_{0}(x)=1+0.5 x ; A(x)=$ 0.00625 (dashed line) and $A(x)=0.005(+0.5 x)$ (solid line); these parameters correspond to the PZT-film with the composition $(1-x) \mathrm{PbTiO}_{3}+x \mathrm{ZrTiO}_{3}, x=0.0-0.3$. 


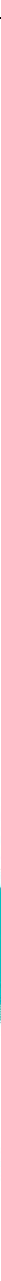




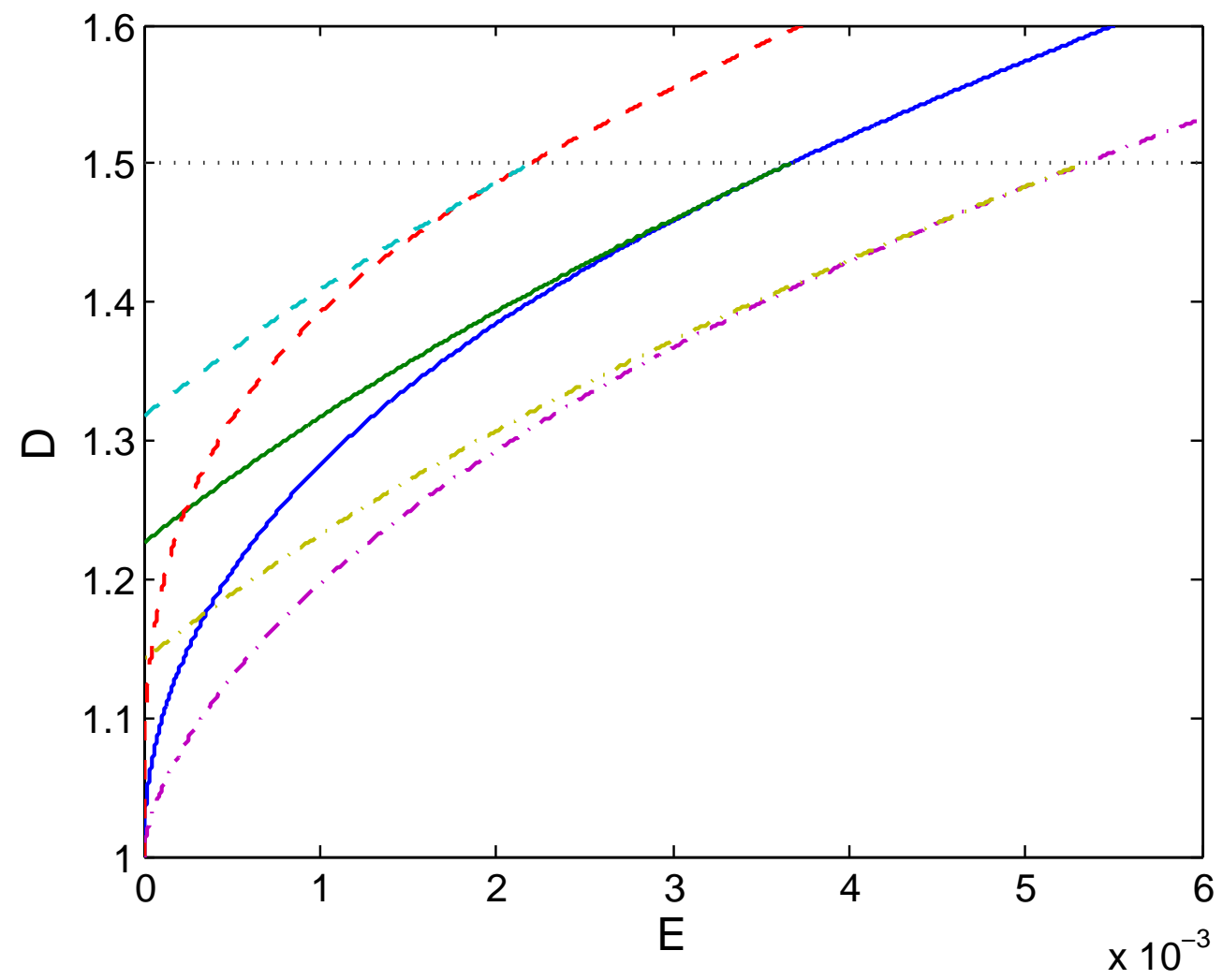


(a)

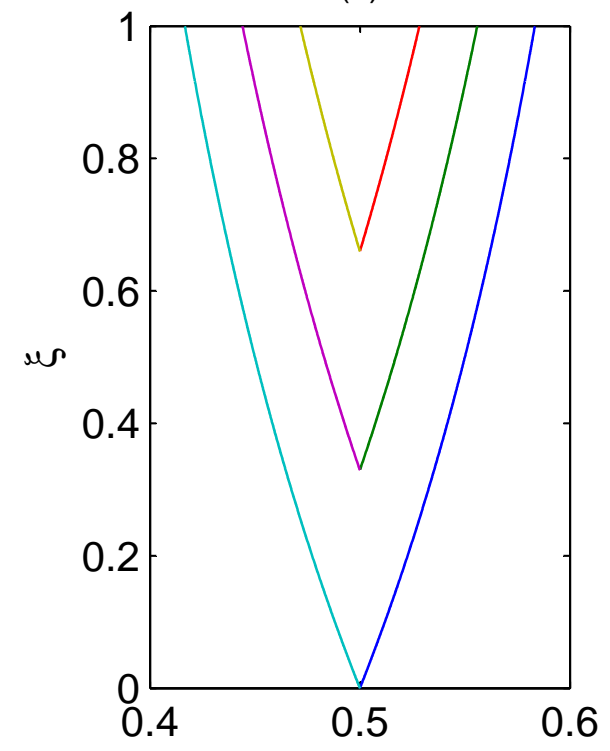

(b)

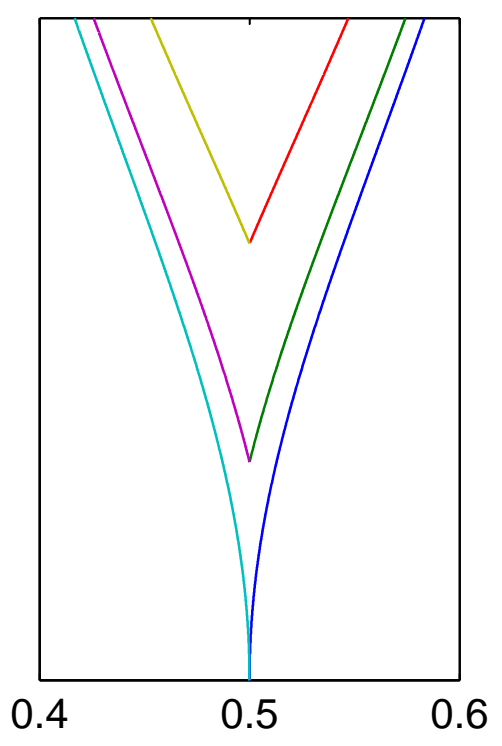

(c)

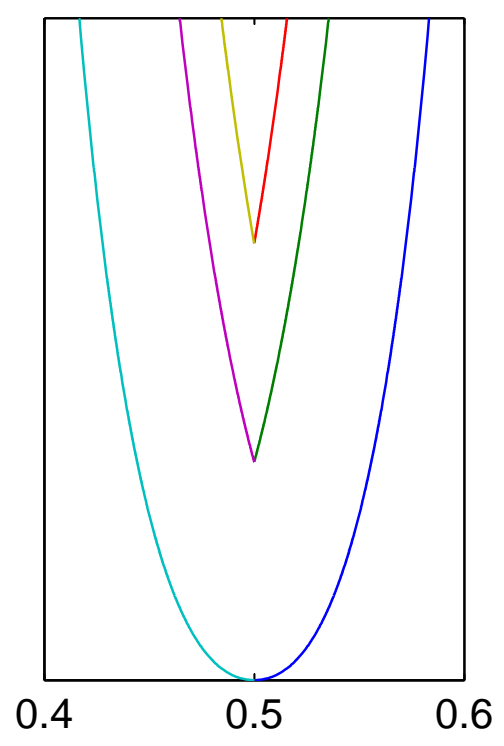




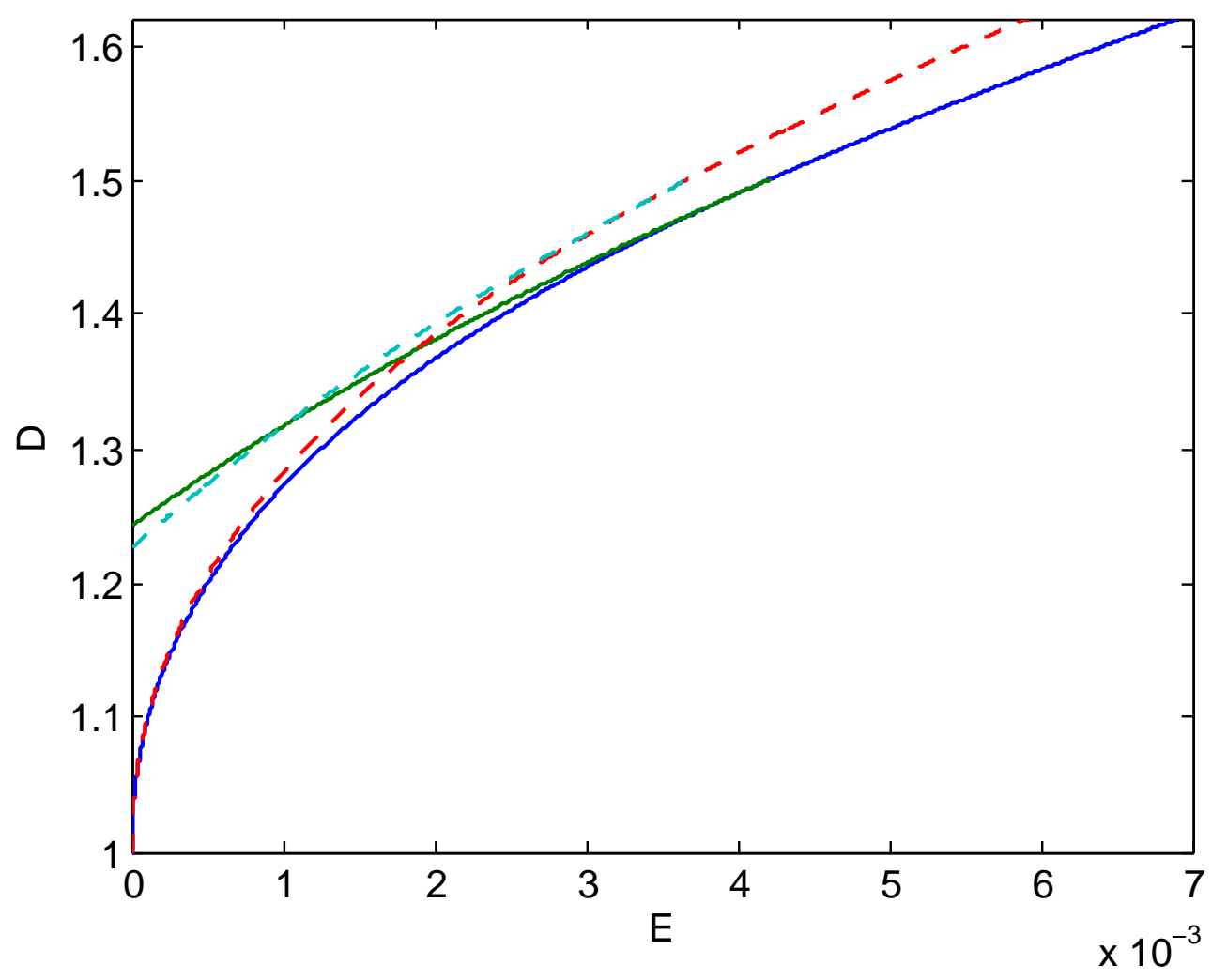

